\title{
Model of the broadband interferometric optical biosensor in a planar configuration
}

\author{
Marek Błahut \\ Department of Optoelectronics, Silesian University of Technology, Akademicka 2A, 44-100 Gliwice
}

Received April 02, 2020; accepted June 28, 2020; published June 30, 2020

\begin{abstract}
The paper presents numerical studies of the model of an optical sensor based on interference of modes in a planar onedimensional step-index configuration, excited by a broadband light source from a selected spectral range. The refractive index variation of measured external surrounding affects the modal properties of a multimode waveguide and spectral field distribution at the output of the structure. The optical system described is designed for analysis of biological substances.
\end{abstract}

In their operation, integrated planar optical waveguide interferometric sensors combine evanescent field sensing and optical phase difference measurement methods [1]. The refractive index variation of external waveguide surrounding influences evanescent field distribution and induces a phase shift of the guided modes. Typically, interference structures are excited from a monochromatic light source by a laser beam. This leads to a sinusoidal dependence of the output signal on the phase difference and for that reasons it is not possible to deduce the direction of the phase changes when the phase difference between two interfering modes is an integer multiple of $\pi$.

The substitution of an input laser light source with a white-light source can solve this problem because the phase change for every wavelength is different and therefore it is possible to deduce from a full spectrum the changes induced by an external region of the waveguide [2].

In this work a novel interferometric optical sensor in a planar configuration is presented, designed to operate with a broadband source. Its optical configuration - the set of refractive indices and the geometry is adjusted to examine biological solutions.

The configuration of a planar interference sensor is shown in Fig.1. The structure consists of a single-mode input waveguide, multimode section which, generally, guides two basic modes and a single-mode output waveguide. The number of excited modes in a multimode waveguide depends on the wavelength - for shorter waves, the number of modes can be higher. The refractive index variation of a measured external surrounding affects the modal properties of excited mode in a different way. The interfering fields of these modes produce an interference signal detected at the sensor's output, whose variations depends on the refractive index change. The structure described is a type of one-dimensional MachZehnder interferometer.

Material parameters refer to the polymer SU-8 waveguide on an $\mathrm{SiO}_{2}$ substrate. Their dispersion characteristics were determined in the paper [3]. SU-8 is suitable as a waveguide layer since it possesses low optical absorption and the fabrication process is fast and technologically easy [4].

It is assumed that the optical system will be used to analyze biological substances. Hence, the suggested refractive index changes of the cover for examined characteristics refer to water solutions and can be expressed as an refractive index of water, which dispersion characteristics are taken from [5], increased by the value $\Delta n_{c}$. It is assumed that $\Delta n_{c}$ is changing within the limits $(0-0.005)$. The structure is excited by the broadband light source from the range of $0.5-0.75 \mu \mathrm{m}$. The image variations and spectral distribution are registered at the output of a single-mode waveguide.

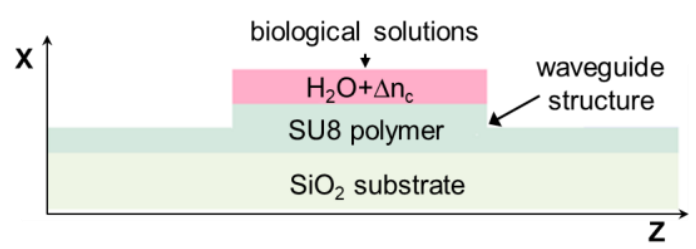

Fig.1. Configuration of the sensor structure.

The thickness of the input and the output waveguides for a single-mode operation amounts to $400 \mathrm{~nm}$ and the thickness $w_{m}$ of a few-mode multimode section is changed from $700 \mathrm{~nm}$ to $900 \mathrm{~nm}$.

The operation principle of the examined optical structure can be determined by modal field analysis. Although the examined multimode structure guides generally only two modes, the derived equations can be used also in the case of a multimode waveguide which guides more modes.

The wave function $\phi_{0}(x)$ of the single-mode waveguide describes the field at the input of a multimode waveguide. This field introduced to the interference section is decomposed into wave fields of all modes of a multimode waveguide: 


$$
E_{M}(x)=\sum_{l=0}^{N-1} c_{l} \cdot \phi_{l}^{M}(x) \text { with } c_{l}=\int_{-\infty}^{+\infty} \phi_{0}(x) \cdot \phi_{l}^{M}(x) d x
$$

where $\phi_{l}{ }^{M}(x)$ are the wave functions of the multimode section of an order $l$ with propagation constants $\beta_{l}$ and excitation coefficients $c_{l}$ defined by overlap integrals.

The field at the distance $z$ is a superposition of all modal fields with different phase shifts:

$$
E_{M}(x, z)=\sum_{l=0}^{N-1} c_{l} \cdot \phi_{l}^{M}(x) \cdot e^{j \beta_{l} \cdot z}
$$

In the next step of the propagation, the field from the end of a multimode waveguide excites the output singlemode waveguide. The field at the output of analyzed structure $E_{\text {out }}$ can be expressed by:

$$
E_{\text {out }}(x, z)=c_{0} \cdot \phi_{0}(x) \cdot e^{j \beta_{0} \cdot z} ; c_{0}=\int_{-\infty}^{+\infty} \phi_{0}(x) \cdot E_{M}(x, L) d x
$$

where $\beta_{0}$ is the propagation constant of a single-mode waveguide, $c_{0}$ describes the overlap integral of both fields and $L$ is the length of a multimode waveguide.

Taking into account Eqs. $(1) \div(3)$, the output power $P_{\text {out }}$ can be easily determined:

$$
P=\left|c_{0}\right|^{2}=\left|\int_{-\infty}^{+\infty} \phi_{0}(x) \cdot \sum_{l=0}^{N-1} \phi_{l}^{M}(x) \cdot e^{-j \beta_{l} \cdot L} d x\right|^{2}
$$

This dependence has been examined numerically as a function of the wavelength for a different geometry of the optical structure.

Optical properties of the examined structure result from modal properties of the waveguides in the assumed spectral range of the broadband source. In Fig. 2 there are presented propagation constants dependences on the wavelengths for a single-mode waveguide of the $400 \mathrm{~nm}$ thickness and multimode of the 700nm and $800 \mathrm{~nm}$ thickness used in numerical simulations for a spectrum range of $0.5 \div 0.8 \mu \mathrm{m}$. Calculations were performed for $\mathrm{TE}$ polarization. It can be seen that the input and output waveguides of the thickness $w=400 \mathrm{~nm}$ are single-mode in the whole range and for a multimode waveguide they are two-mode - for $w_{m}=700 \mathrm{~nm}$ within the range $(0.5-$ $0.7 \mu \mathrm{m})$ and for $w_{m}=800 \mathrm{~nm}$ within the range $(0.5-$ $0.795 \mu \mathrm{m})$.

Figure 3a shows typical output spectral characteristics $P(\lambda)$ of the structure covered by water. The length of the analyzed two-mode section $L$ is equal to $2000 \mu \mathrm{m}$. The length of input and output waveguides amounts to $5000 \mu \mathrm{m}$.

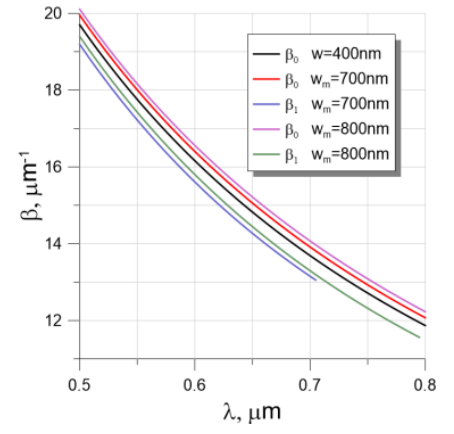

Fig.2. Propagation constants dependence on the wavelength.

Figure $3 \mathrm{~b}$ presents the propagation constants difference of two basic modes as a function of the wavelength for different values of the refractive index of the cover.
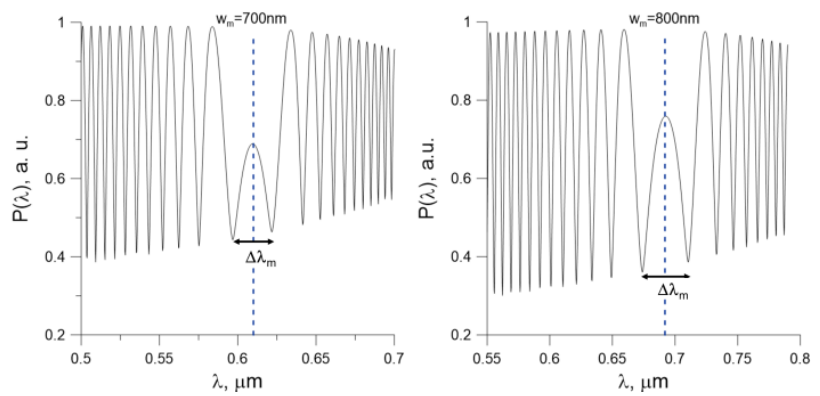

(a)
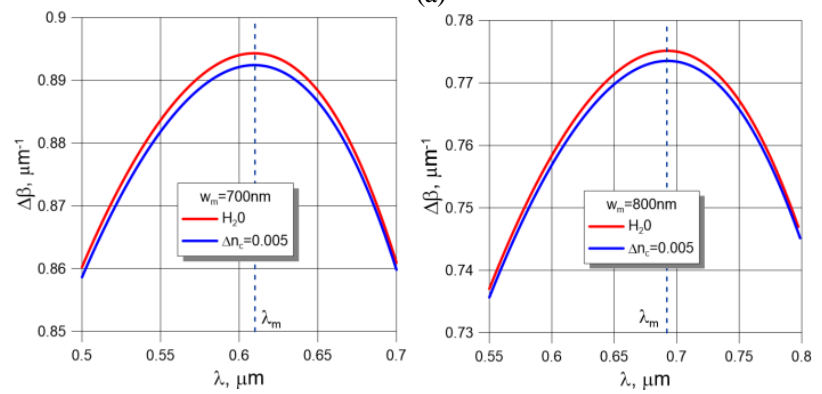

(b)

Fig. 3. Output spectral characteristics (a) and propagation constants difference of two basic modes as a function of wavelength (b).

One can see characteristic effects which occur near the wavelengths shown in Fig. 3a by vertical lines. This wavelengths, as it is shown in Fig. 3b, define the spectral ranges $\Delta \lambda_{m}$ (of the width of $\sim 0.03 \mu \mathrm{m}$ ) near the maximum values $\lambda_{m}$ inside of which the propagation constants difference of modes and connected phase of the signal change very slowly with the wavelength, for the assumed geometry of the structure. This characteristic maximum value does not depend on the refractive index of the cover and shifts toward longer waves together with waveguide thickness. This property makes it possible to fit the optimal waveguide geometry to an input source spectral range.

Figure $4 \mathrm{a} \div \mathrm{b}$ shows an optical structure response to refractive index variations of the surroundings. It is assumed that a two-mode waveguide length amounts to 
2000nm A spectral characteristics shift is observed with refractive index changes, which is the strongest in the vicinity of the spectral range $\Delta \lambda_{m}$.

(a)

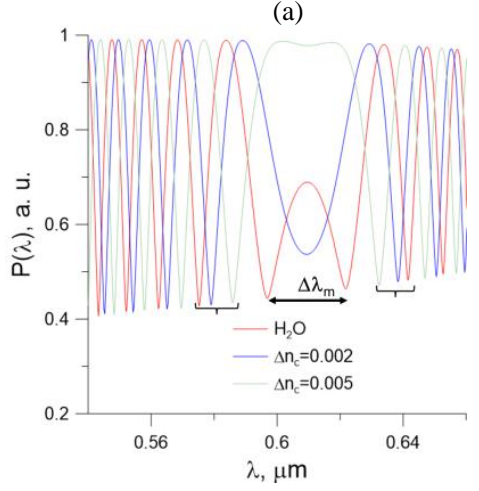

(b)

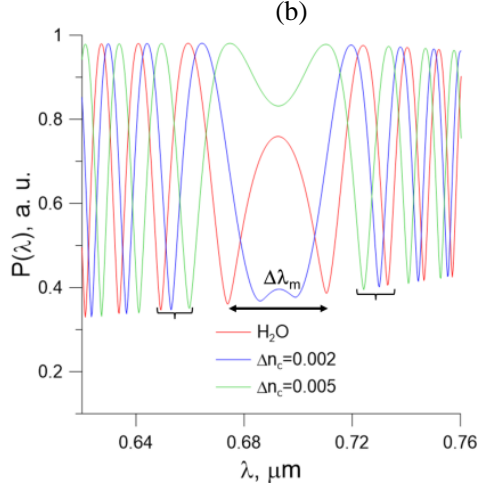

Fig. 4. Spectral characteristics $P(\lambda)$ for different values of the refractive index of the cover for the waveguide thickness $w_{m}$ equal to a) $700 \mathrm{~nm}$ and b) $800 \mathrm{~nm}$.

For the waves of shorter wavelengths, on the left sight from $\Delta \lambda_{m}$, the signal extremes shift towards longer waves with the increase of the refractive index of the cover. On the other hand, for the waves of longer wavelengths, on the right sight from $\Delta \lambda_{m}$, the signal extremes shift towards shorter waves with an increase in the refractive index of the cover. Similar effects have been observed in the case of broadband differential interferometers [3].

Figure 5 presents the dependence of spectral characteristics $\mathrm{P}(\lambda)$ on the two-mode waveguide length $L$. Calculations were performed for the waveguide of the thickness $w_{m}=800 \mathrm{~nm}$ and two different values of $L$ $2000 \mu \mathrm{m}$ and $10000 \mu \mathrm{m}$. As the waveguide length increases, the number of extrema in the examined wavelength range grows but the width of $\Delta \lambda_{m}$ decreases. The change of the length $L$ affects the extrema locations and their shifts.

The impact of refractive index variations on the spectral distribution change is strongest near the range $\Delta \lambda_{m}$. The maxima on both sides of $\Delta \lambda_{m}$ shift towards each other. Taking into account this feature, it is possible to construct one of possible operating characteristics of the sensor, by measuring the difference of mutual shifts of extremes $\Delta d$, closest to the range $\Delta \lambda_{m}$ for different values of the refractive index of the cover. (a)

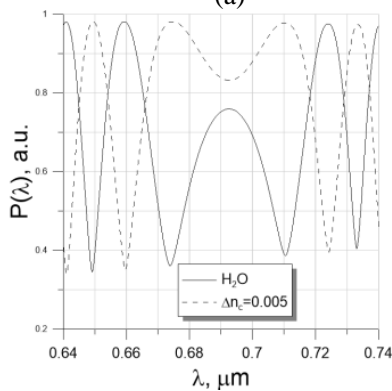

(b)

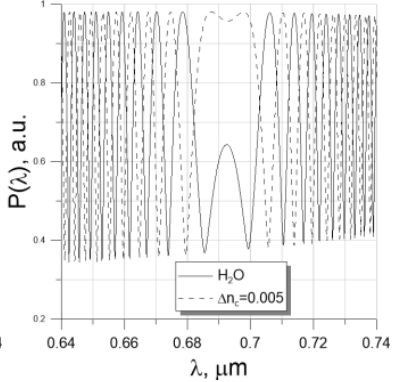

Fig. 5. Spectral characteristics $P(\lambda)$ for the multimode waveguide $L$ equal to a) $2000 \mu \mathrm{m}$ and b) $10000 \mu \mathrm{m}$.

Dependences determined in that way are presented in Figs. $6 \mathrm{a} \div \mathrm{b}$. Extremes used to determine this operating characteristic are marked in Figs. $4 a \div b$ by brackets. (a)

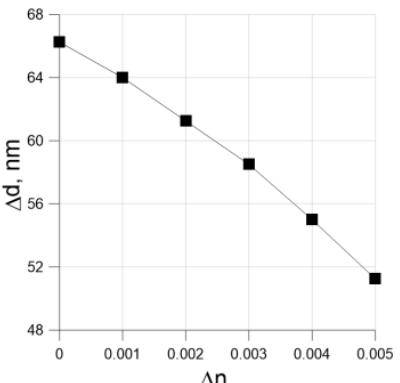

(b)

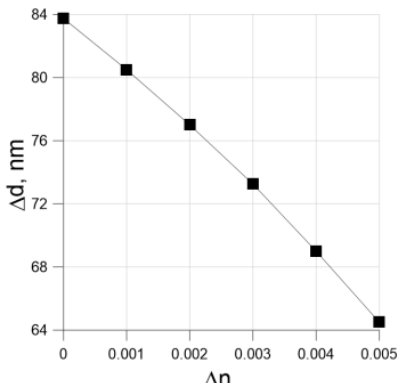

Fig. 6. Difference of mutual shifts of extremes $\Delta d$ as a function of refractive index of the cover changes in relation to the water for the waveguide thickness $w_{m}$ equal to a) $700 \mathrm{~nm}$ and b) $800 \mathrm{~nm}$

Taking into account wavelength accuracy of spectral analyzers, e.g. [6], the refractive index changes of water solutions can be measured with an accuracy below $10^{-5}$.

The configuration proposed makes it possible to determine refractive index variations from the shifts of output signal spectral distribution instead of measurements of output power changes. For that reason, the method is independent of the excitation level at the input.

\section{References}

[1] M. Blahut, Proc. SPIE 10455, 1045503 ( 2017).

[2] K. Misiakos, et al., Opt. Express 22, 8856 (2014).

[3] K. Gut, Nanomaterials 9, 729 (2019).

[4] M. Nordstrom, et al., J. Light. Techn. 25, 1284 (2007).

[5] D. Segelstein, M.S. Thesis (University of Missouri, 1981).

[6] https://www.yokogawa.com/pl/solutions/products-platforms/ 